Research Square

\title{
Increases of Phospho-Tau (Ser202/Thr205) in the Olfactory Regions Are Associated With Impaired EEG and Olfactory Behavior in Traumatic Brain Injury Mice
}

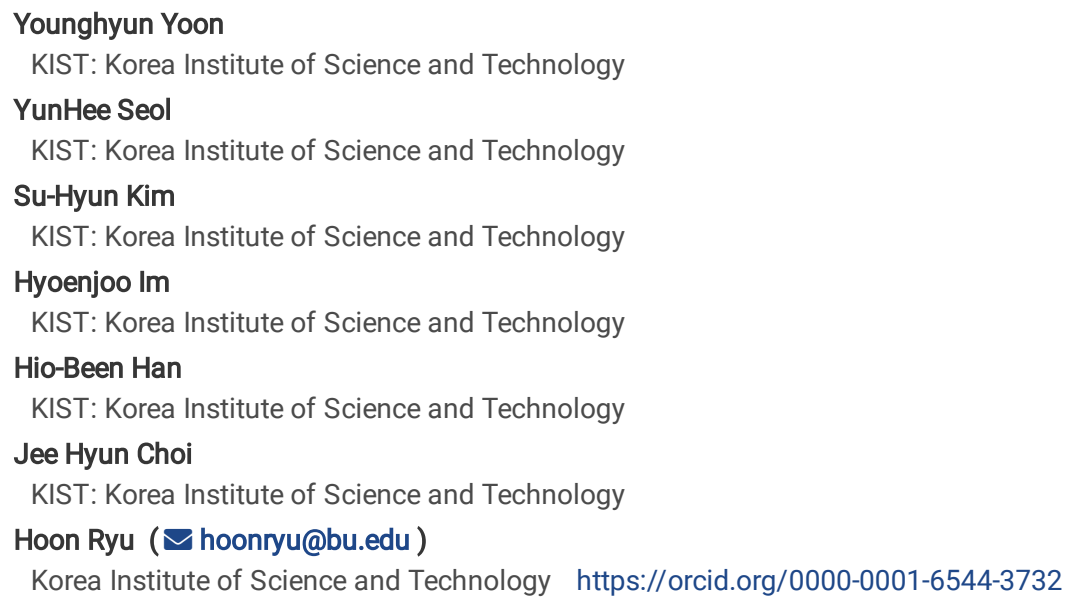

\section{Research Article}

Keywords: traumatic brain injury (TBI), olfactory system, electroencephalogram (EEG), tauopathy, neuronal atrophy

Posted Date: September 23rd, 2021

DOI: https://doi.org/10.21203/rs.3.rs-208312/v2

License: () (1) This work is licensed under a Creative Commons Attribution 4.0 International License. Read Full License 


\section{Abstract}

Traumatic brain injury (TBI) leads to long-term cognitive impairments with an increased risk for neurodegenerative and psychiatric disorders. Among these various impairments, olfactory dysfunction is one of the most common symptoms in TBI patients. However, there are very few studies that show the association between olfactory dysfunction and repetitive TBI, which accounts for the majority of all head injuries. In this present study, we investigated the effects of repetitive TBI on olfactory functioning and the related pathological neuronal injuries in mice. Through pathological examination, we found a significant increase in p-Tau (S202/T205) in olfactory bulb-associated areas such as the Anterior Olfactory Nucleus (AON), Pyriform Cortex (PC), Tenia Tecta (TT), and Olfactory Tubercle (OT). Neuronal atrophy in the Lateral Anterior Olfactory Nucleus (AOL), Granule Layer Olfactory Bulb (GrO), and Dorsal Tenia Tecta (DTT) was also found to be correlated with p-Tau levels. Furthermore, electroencephalography (EEG) of repetitive TBI mouse models showed impaired spontaneous delta oscillation, as well as altered cross-frequency coupling between delta phase and amplitudes of the fast oscillations in the resting-state olfactory bulb. Also, abnormal alterations in EEG band powers were observed during the olfactory oddball paradigm test. This study provides evidence of pathological and physiological alterations in the mouse olfactory system caused by repetitive TBI. Together, $\mathrm{p}$-Tau alterations and EEG impairments may serve as important biomarkers of olfactory track-associated dysfunctions and behavioral impairments commonly observed in repetitive TBI patients.

\section{Key Message}

- Repetitive TBI affected olfactory function and induced molecular pathological neuronal injuries in mice

- -Tau (S202/T205) levels were significantly increased in olfactory bulb-associated areas

- An increase of neuronal atrophy in the olfactory bulb-associated areas was correlated with an increase of $p$-Tau levels

- TBI impaired spontaneous delta oscillation in the resting-state olfactory bulb

- $\mathrm{p}$-Tau alterations and EEG impairments may serve as important biomarkers of olfactory track-associated dysfunctions in TBI

\section{Introduction}

Traumatic brain injury (TBI) accounts for approximately 1.7 million patients yearly in the United States and remains as a leading cause of mortality worldwide [1-3]. Dep ending on the nature and severity of TBI, anatomical changes are highly variable and often result in long-term neurobehavioral and neuropathological sequelae [4-6]. Clinical manifestations may include neuropsychiatric disturbances such as memory impairment, and difficulty sleeping. As well as mood or behavioral abnormalities such as depression, aggression, and anxiety [7].

Out of the many pathological phenotypes, olfactory dysfunction is often an early signal for chronic neurodegenerative disorders such as chronic traumatic encephalopathy (CTE), Alzheimer's disease (AD), and Parkinson's disease [4, 6, 8, 9]. For instance, pathophysiological markers such as a-synuclein and tauopathy have been linked to changes in the olfactory system and are well known to be indicators of dementia risk. In the case of Parkinson's disease, the olfactory bulb has been proposed as a possible induction site for Lewy pathology. Pathological changes were also observed in other areas of the olfactory system such as the anterior olfactory nucleus (AON), cortical nucleus of the amygdala, piriform cortex, olfactory tubercle, entorhinal cortex, and orbitofrontal cortex [10].

While the associations between olfactory dysfunction and its pathological markers have been mainly observed in cases of moderate-to-severe TBI, in recent years, special interest has headed towards those of repetitive sub-concussions or repetitive mild-TBI (mTBI) [11-16]. However, a gap in TBI studies has been the use of a simple model system that accurately reads the physiological and behavioral changes of olfactory disruption after mTBI events [6]. Human imaging tools, such as MRI and CT scans, have limitations in the sensitivity of detecting focal brain lesions caused by TBI, which often result in mTBI patients showing cognitive deficits even when MRI and CT results are normal [17]. Furthermore, Callaham \& Hinkebein found that $65 \%$ of TBI patients experienced anosmia, although most were unaware of their impaired olfaction. Anosmia was also correlated with greater cognitive disability [4].

These subtle behavioral differences in olfactory dysfunction show that a sensitive measuring tool is needed to detect TBI related injuries in human patients. Thus, we used a proper mouse model that closely imitates the conditions of human TBI patients. Past TBI models have utilized invasive methods such as controlled cortical injury (CCI), blast-induced TBI (bTBI), fluid percussion injury (FPI), and closed/head impact model of engineered rotational acceleration (CHIMERA). These methods are likely to result in fractures, brain contusions, or anoxic-ischemic injuries which lead to significant neurological impairments and high mortality rates. Human patients who experience repetitive TBI, rarely undergo acute injuries, which make these animal models inappropriate to use to examine the post-injury conditions of human patients.

In this study, we produced repetitive TBI mice with repetitive concussion to emulate human sub concussion scenarios and examined the various neurological consequences of repetitive head injuries. Although the buried food-seeking test is the most reliable olfactory assay in rodents so far [18, 19], it is reported to be closely associated with other cognitive deficits, such as abnormalities in food motivation or elevated fear. For this reason, it is hard to detect and measure subtle olfactory impairments may remain undetected [20].

To overcome this weakness, we used electroencephalogram (EEG) to measure neuronal oscillations of mice during an olfactory oddball paradigm test to scale olfactory functionality instead. Then, to avoid disruption from surgery, a separate mouse cohort was used to examine the neurological consequences of the olfactory system in repetitive TBI mice. Using an interdisciplinary approach, we found alterations in olfaction-related EEG activities and found pathophysiological signs of neuronal atrophy such as tauopathy and cell shrinkage in olfactory related areas.

\section{Materials And Methods}




\section{Mice}

A total of 35 mice were used for this study. 14 Thy1 mice (B6.Cg-Tg(Thy1-COP4/EYFP)18Gfng/J, Stock \#007612, The Jackson Laboratory, Bar Harbor, ME, USA; 9-16 weeks; body weight 19-31g) of both genders (male, $n=4$; female, $n=10$ ) and 21 male wild type mice (C57BL/6N; 8-10 weeks) were used. All mice received five weight drop-induced closed diffuse TBI events. While Thy 1 mice were used to collect odor-evoked electrophysiology data, 10 wild type mice were used to collect immunochemistry data and 11 wild type mice were used to collect Y-maze behavioral data. All mice were born and raised under standard laboratory conditions of a 12-hour light/dark cycle at $22^{\circ} \mathrm{C}$ with $55 \%$ humidity and were fed a standard laboratory diet and water ad libitum.

\section{Repetitive concussion treatment}

Mice received a total of five weight drop-induced closed diffuse TBls, each administered every three days. Closed diffuse TBls were performed by a weightdrop device (weight $100 \mathrm{~g}$, fall height $75 \mathrm{~cm}$, angle $90 \mathrm{deg}$ ), as previously described with a slight modification [21, 22]. Control and TBI mice were anesthetized with $2 \%$ Avertine $(23 \mu \mathrm{g} / \mathrm{g})$ IP injection before receiving the weight drop-induced TBI. Sham-injured animals were subjected to the same protocol, but no mass was dropped (Fig. 1). No mice were killed following impact, and mice were monitored for signs of severe injury or skull fracture.

\section{Immunohistochemistry}

Immunohistochemistry was performed as previously described [22]. Animals were deeply anesthetized with $2 \%$ avertine ( $23 \mu \mathrm{g} / \mathrm{g}$, i.p.) and then perfused through the ascending aorta with $100 \mathrm{ml}$ of cold $0.1 \mathrm{M}$ phosphate buffer saline (PBS), followed by $100 \mathrm{ml}$ of $4 \%$ paraformaldehyde in $0.1 \mathrm{M}$ PBS. The brain was removed, post-fixed overnight and cryoprotected in $30 \%$ sucrose in $0.1 \mathrm{M} \mathrm{PBS}$ at $4^{\circ} \mathrm{C}$. Then cut by cryotome into $30 \mu \mathrm{m}$ coronal sections, and processed histochemically as free-floating sections. Tissue sections were then rehydrated, blocked with blocking solution (3\% $\mathrm{H} 2 \mathrm{O} 2)$, and incubated with $\mathrm{p}$-Tau (S202/T205) (1:200; Thermofisher, Waltham, MA, USA) and p-Tau (S199) (1:200; Abcam, Cambridge, MA, USA) for 24 hrs. After three times of washing, the slides were processed with Vector ABC Kit (Vector Lab, Burlingame, CA, USA). Immunoreactive signals were developed with DAB chromogen (Thermo Fisher Scientific, Waltham, MA, USA). Slides were photomicrographed under bright field microscopy and analyzed using Image J.

\section{Electrode implantation}

EEG electrodes were implanted after repetitive concussion treatment. Mice were deeply anesthetized with ketamine (120mg/kg, intraperitoneal) and xylazine $(6 \mathrm{mg} / \mathrm{kg}$, intraperitoneal), and fixed in a stereotaxic apparatus (David Kopf Instruments, Model 902, Tujunga, CA, USA). Sterilized micro-screw electrodes (Asia Bolt, South Korea) were fixed onto the skull surface of the olfactory bulb (anteroposterior, $4.8 \mathrm{~mm}$; mediolateral, $1.2 \mathrm{~mm}$; dorsoventral, $-1.1 \mathrm{~mm}$ from bregma), frontal (anteroposterior, $0.5 \mathrm{~mm}$; mediolateral, $1.2 \mathrm{~mm}$ ), and parietal cortex (anteroposterior, $-3.08 \mathrm{~mm}$; mediolateral, $3.75 \mathrm{~mm}$ ), with ground/reference electrodes implanted on the interparietal bone. The electrode coordinates were selected in accordance with the mouse atlas [23]. To secure the electrode positions, dental cement (VertexTM Self-Curing, Vertex-Dental, Netherlands) was applied, along with two polycarbonate nuts (inner diameter 3 mm, Nippon Chemi-Con, Japan) which were attached to the caudal edge of the cement for head-fixation during the experiment.

\section{LFP recording}

After one week of recovery period, mice were placed in front of the olfactometer while being head fixed in a custom mouse restrainer. Mice were placed so that their nose tip had a $1 \mathrm{~cm}$ distance from the olfactometer outlet. Three-channel LFP (the olfactory bulb, the frontal cortex, and the parietal cortex) data were collected during the olfactory oddball paradigm with a Cerebus amplifier (Blackrock Microsystems, UT, USA). All signals were digitized with a 2 kHz sampling rate and bandpass filtered from 0.3 to $500 \mathrm{~Hz}$.

\section{Olfactory Oddball Paradigm}

The experiment was conducted utilizing the olfactometer setup previously described by Kum et al. (2019) [20] (Fig. 1c). For odor delivery, constant flow of filtered air (1L/min) was delivered, with odor stimuli being diluted by pumped air $(200 \mathrm{ml} / \mathrm{min})$. Methyl salicylate (Sigma Aldrich, > $99 \%$ purity, mineral oil solution, odorant:solvent ratio was 3:1) and ethyl acetate (Sigma Aldrich, >99.5\% purity, distilled water solution, odorant:solvent ratio was 1:1) were selected for the olfactory oddball paradigm; methyl salicylate being the standard odorant and ethyl acetate being the deviant odorant. The standard and deviant odor were presented randomly with a 5:1 ratio. The stimulation period was 2 seconds with an inter-stimulus interval of 20 seconds. After presented, odor stimuli were vacated through the vacuum pump (1.5L/min) (Fig. 1d).

One session was composed of 90 trials (75 standard trials and 15 deviant trials), lasting approximately 33 minutes (Fig. $1 \mathrm{~d}$ ). Each mouse received four sessions of experiment in total, which were divided into two days in order to prevent adaptation to the odor stimuli and over-exhaustion caused by the long duration of head-fixing. Two sessions were given each day with an interval of 30 minutes between each session, and there were three to five days between the two days of experiment. The recording room was ventilated for 30 minutes after each recording session.

\section{LFP analysis}

The LFP data were processed in Matlab 2019a (Mathworks, MA, USA) with Signal Processing Toolbox (ver. 8.2). First, the signals were bandpass-filtered (1$150 \mathrm{~Hz}$ using a 5-th order Butterworth filter, then transformed into time-frequency domain using sliding Hanning window (512 ms length, $100 \mathrm{~ms}$ resolution) and fast Fourier transform to obtain amplitude spectrogram. The frequency bands of interest were delta $(1.5-4 \mathrm{~Hz})$, theta $(4-8 \mathrm{~Hz})$, beta $(12-30 \mathrm{~Hz})$, low gamma $(40-60 \mathrm{~Hz})$, and high gamma $(70-120 \mathrm{~Hz})$ bands. In case of analysis of resting state, the amplitude spectrum of each channel was obtained from each individual by averaging the spectrogram of 2-min before the olfactory oddball paradigm. Cross-frequency coupling was evaluated using modulation index (MI), which is a statistical measure of phase lock of fast oscillation amplitudes to the relative time window of slow oscillation [24]. The MI 
was calculated by dividing the Kullback-Leibler distance of actual amplitude distribution of fast oscillations over the phase of slow oscillations (bin number $=30$ ) with uniform distribution. For the analysis of olfactory oddball paradigm, event-related spectral perturbation (ERSP) was obtained by averaging the spectrogram of baseline-corrected signals with temporal and frequency resolutions of $100 \mathrm{~ms}$ and $2 \mathrm{~Hz}$. To match the number of trials between standard and deviant trials, only the standard trials right before the deviant trials were used. To evaluate the diminished oscillatory activities in TBI model mice, independent sample t-tests (alpha $=0.05$, one-tailed) were performed using ttest 2 . $\mathrm{m}$ function in Matlab 2019a, over the individual mean value in the band-of interests and time-of-interests (degree of freedom $=15$ ).

\section{Olfactory Discrimination using Y-maze}

Y maze was performed the subsequent day after the fifth weight drop, PND (12 weeks) as previously described with a modification [9]. The Y maze apparatus was composed of three enclosed arms $(35.3 \times 6 \times 18.4 \mathrm{~cm}(\mathrm{I} \times \mathrm{w} \times \mathrm{h}))$ constructed of white acrylic. A tray of home bedding $(7.2 \times 2.9 \times 1.3)$ was placed at the end of one arm, and the same to its adjacent arm with new bedding. Each trial began by placing each individual mouse in the arm with no bedding and was observed for 10 minutes. Total distance $(\mathrm{cm})$, velocity $(\mathrm{cm} / \mathrm{s})$, time (s), frequency (count), and latency to start (time) at each arm was recorded by Ethovision Software (Noldus Information Technology, Wageningen, Netherlands).

\section{Results}

\section{Olfactory bulb associated areas exhibit increased tauopathy in repetitive TBI}

To examine whether short-term TBI is associated with elevated tauopathy, we first performed immunohistochemistry. As expected, the immunoreactivity of tau was strongly elevated in olfactory bulb associated areas in TBI mice ( $N=5)$ compared to control subjects $(\mathrm{N}=5$; Fig. 2a). Interestingly, an increase in tauopathy was not observed in the Main Olfactory Bulb. However, densitometry analysis showed a significant increase in tauopathy in six out of nine regions examined including the Anterior Olfactory Nucleus (lateral AOL; medial AOM), Olfactory Bulb (GrO), Tenia Tecta (dorsal DTT), Pyriform Cortex, and Olfactory Tubercle.

\section{Tauopathy is correlated with neuronal atrophy in repetitive TBI}

To better understand the influence of tauopathy to neuronal atrophy or cell size shrinkage in olfactory bulb associated areas, we performed densitometry analysis combined with cell size analysis on the regions displaying the strongest tauopathy. Densitometry analysis verified the AOL, AOM, GrO, and DTT or the AON and TT regions (Fig. 3a) as the areas with the most significant increases in tauopathy, in controls ( $N=5)$ versus repetitive TBI subjects ( $N=5$; Fig. $3 b$ ). Additionally, cell size analysis of the same cells measured for densitometry revealed cell shrinkage in three of the four areas observed, including the AOL, GrO, and DTT (Fig. 3c).

\section{Altered spectral power of slow oscillations of olfactory bulb in concussed mice}

We investigated the effects of repetitive TBI on spontaneous oscillation by measuring LFP in the prefrontal cortex, hippocampus, and olfactory bulb. The level of spontaneous oscillations was quantified with power spectral analysis and is summarized in Table 1. Compared to the control group ( $\mathrm{N}=8$ ), mice in the concussed group $(\mathrm{N}=9$ ) exhibited a significant decrease in delta bands of the olfactory bulb whereas spontaneous oscillations in the prefrontal cortex and hippocampus were not affected in the concussed group (Fig. 4a-b).

\section{Table1. Summary of spontaneous oscillation and modulation by delta phase}

Results of analysis of resting state EEG. Independent t-test was used. The values are average and standard deviation in parenthesis.

We further investigated the cross-frequency coupling by calculating the modulation index (MI) according to Tort, Komorowski, Eichenbaum, \& Kopell, 2010 [24]. We calculated Ml for delta and theta phases. We found that MI values for the delta phase were significantly attenuated in fast oscillations of the olfactory bulb of repetitive m-TBI mice. The comodulograms (Fig. 4c) display the averaged MI across animals for the control mice and for the concussed mice, with differences largely captured by changes in scale. Statistical tests returned that the cross-frequency couplings between delta phase and beta/low gamma amplitude are significantly reduced in the concussed model (Fig. 4d).

\section{Attenuated response to deviants in concussed mice}

We performed an olfactory oddball paradigm with methyl salicylate as a standard odor and ethyl acetate as a deviant odor. Fig. 5a shows the averaged ERSP for standard and deviant stimuli. Overall, a broadband response was elicited by odor release but beta oscillation responded in the most eminent manner. As expected, the deviant stimuli elicited stronger in a significant way. Fig. 5b shows the grand average of differential spectrograms of ERSP calculated from individual mice, showing an overall debilitation. Fig. $5 \mathrm{c}$-d marks the ranges of frequency showing the significant or marginal differences in responses to the deviant odor in the concussed mice $(\mathrm{N}=9)$ compared to the control mice $(\mathrm{N}=8)$. The responses in the measured areas are summarized in Table 2. It is noteworthy that the relative power of dominant power (beta) dropped from 65\% (control) to $35 \%$ (TBI), but in a marginally significant way. On the other hand, the other frequency EEG bands were enhanced in a significant way by repetitive head injury as summarized in Table 2.

\section{Table 2. Summary of event spectral density during olfactory oddball paradigm.}

The ratio, $\left(\mathrm{P}_{\text {deviant }}-\mathrm{P}_{\text {standard }}\right) / \mathrm{P}_{\text {standard }}$ were calculated for each frequency band and then noted in percent, where $\mathrm{P}_{\text {standard }}$ and $\mathrm{P}_{\text {deviant }}$ are powers during standard and deviant odor stimulations, respectively. 


\begin{tabular}{|c|c|c|c|c|c|c|c|c|c|c|c|c|c|}
\hline \multirow{2}{*}{$\begin{array}{l}\text { EEG } \\
\text { Parameters }\end{array}$} & \multirow{2}{*}{$\begin{array}{l}\text { Frequency } \\
\text { bands }\end{array}$} & \multicolumn{4}{|c|}{ Frontal Cortex } & \multicolumn{4}{|c|}{ Parietal Cortex } & \multicolumn{4}{|c|}{ Olfactory Bulb } \\
\hline & & $\begin{array}{l}\text { Control } \\
(n=8)\end{array}$ & $\begin{array}{l}\text { TBI } \\
(n=9)\end{array}$ & $\begin{array}{l}t \text {-stat } \\
(d f=15)\end{array}$ & $\begin{array}{l}p- \\
\text { value }\end{array}$ & $\begin{array}{l}\text { Control } \\
(n=8)\end{array}$ & $\begin{array}{l}\text { TBI } \\
(n=9)\end{array}$ & $\begin{array}{l}t \text {-stat } \\
(d f=15)\end{array}$ & $\begin{array}{l}p- \\
\text { value }\end{array}$ & $\begin{array}{l}\text { Control } \\
(n=8)\end{array}$ & $\begin{array}{l}\text { TBI } \\
(n=9)\end{array}$ & $\begin{array}{l}t \text {-stat } \\
(d f=15)\end{array}$ & $\begin{array}{l}p- \\
\text { value }\end{array}$ \\
\hline \multirow{10}{*}{$\begin{array}{l}\text { Amplitude } \\
(\mathrm{mV})\end{array}$} & \multirow[t]{2}{*}{ Delta } & 15.21 & 14.66 & \multirow[t]{2}{*}{0.52} & \multirow[t]{2}{*}{0.3056} & 12.99 & 13.85 & \multirow[t]{2}{*}{-0.72} & \multirow[t]{2}{*}{0.2425} & 23.30 & 17.52 & \multirow[t]{2}{*}{2.02} & \multirow[t]{2}{*}{0.0309} \\
\hline & & (1.99) & (2.33) & & & $(2.73)$ & $(2.23)$ & & & $(6.79)$ & $(4.99)$ & & \\
\hline & \multirow[t]{2}{*}{ Theta } & 16.40 & 15.81 & \multirow[t]{2}{*}{0.57} & \multirow[t]{2}{*}{0.2898} & 15.49 & 16.05 & \multirow[t]{2}{*}{-0.33} & \multirow[t]{2}{*}{0.3718} & 17.91 & 15.77 & \multirow[t]{2}{*}{1.33} & \multirow[t]{2}{*}{0.1013} \\
\hline & & $(2.20)$ & (2.04) & & & $(3.05)$ & $(3.85)$ & & & $(3.46)$ & (3.17) & & \\
\hline & \multirow[t]{2}{*}{ Beta } & 5.11 & 4.70 & \multirow[t]{2}{*}{1.28} & \multirow[t]{2}{*}{0.1100} & 4.80 & 4.77 & \multirow[t]{2}{*}{0.07} & \multirow[t]{2}{*}{0.4708} & 5.12 & 4.37 & \multirow[t]{2}{*}{2.05} & \multirow[t]{2}{*}{0.0293} \\
\hline & & $(0.80)$ & $(0.51)$ & & & $(0.74)$ & $(0.80)$ & & & $(1.04)$ & $(0.37)$ & & \\
\hline & Low & 2.59 & 2.39 & 1.17 & 0.1311 & 2.50 & 2.47 & 0.21 & 0.4191 & 2.88 & 2.73 & 0.69 & 0.2492 \\
\hline & & $(0.44)$ & $(0.24)$ & & & $(0.44)$ & $(0.24)$ & & & $(0.56)$ & $(0.35)$ & & \\
\hline & High & 1.52 & 1.48 & 0.26 & 0.4004 & 1.44 & 1.48 & -0.27 & 0.3957 & 1.99 & 1.97 & 0.11 & 0.4586 \\
\hline & & $(0.36)$ & $(0.23)$ & & & $(0.39)$ & $(0.19)$ & & & $(0.44)$ & $(0.29)$ & & \\
\hline Coupling to & Beta & 0.54 & 0.96 & -1.89 & 0.0388 & 1.50 & 1.71 & -0.69 & 0.2500 & 5.68 & 1.71 & 2.73 & 0.0077 \\
\hline & & $(0.35)$ & $(0.54)$ & & & $(0.67)$ & $(0.62)$ & & & $(4.32)$ & $(0.65)$ & & \\
\hline 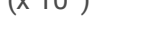 & Low & 0.49 & 0.86 & -1.88 & 0.0396 & 0.89 & 1.10 & -0.76 & 0.2284 & 4.73 & 2.53 & 2.05 & 0.0289 \\
\hline & 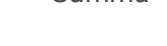 & $(0.38)$ & $(0.43)$ & & & $(0.74)$ & $(0.35)$ & & & $(2.83)$ & $(1.42)$ & & \\
\hline & High & 0.72 & 1.23 & -1.94 & 0.0360 & 0.61 & 1.36 & -2.18 & 0.0227 & 7.22 & 7.11 & 0.03 & 0.4884 \\
\hline & & $(0.53)$ & $(0.55)$ & & & $(0.46)$ & $(0.86)$ & & & (6.18) & $(8.86)$ & & \\
\hline EEG & Frequency & Frontal Cor & ortex & & & Parietal Co & ortex & & & Olfactory & Bulb & & \\
\hline & bands & $\begin{array}{l}\text { Control } \\
(n=8)\end{array}$ & $\begin{array}{l}\text { TBI } \\
(n=9)\end{array}$ & $\begin{array}{l}t \text {-stat } \\
(d f=15)\end{array}$ & $\begin{array}{l}p- \\
\text { value }\end{array}$ & $\begin{array}{l}\text { Control } \\
(n=8)\end{array}$ & $\begin{array}{l}\text { TBI } \\
(n=9)\end{array}$ & $\begin{array}{l}t \text {-stat } \\
(d f=15)\end{array}$ & $\begin{array}{l}p- \\
\text { value }\end{array}$ & $\begin{array}{l}\text { Control } \\
(n=8)\end{array}$ & $\begin{array}{l}\text { TBI } \\
(n=9)\end{array}$ & $\begin{array}{l}t \text {-stat } \\
(d f=15)\end{array}$ & $\begin{array}{l}p- \\
\text { value }\end{array}$ \\
\hline Amplitude & Delta & -1.62 & 1.60 & -0.49 & 0.3146 & -15.94 & -10.13 & -1.40 & 0.0915 & -7.38 & 9.49 & -2.36 & 0.0160 \\
\hline (\%) & & (9.99) & (15.87) & & & $(3.90)$ & (11.15) & & & (17.07) & (12.22) & & \\
\hline & Theta & -4.26 & 2.70 & -2.09 & 0.0272 & -8.55 & -1.48 & -2.58 & 0.0105 & 7.69 & 20.43 & -2.56 & 0.0109 \\
\hline & & $(4.96)$ & $(8.17)$ & & & $(2.41)$ & (7.39) & & & (13.26) & $(6.56)$ & & \\
\hline & Beta & 2.95 & 7.75 & -0.96 & 0.1751 & 3.04 & 8.70 & -0.92 & 0.1851 & 64.85 & 35.49 & 1.34 & 0.0985 \\
\hline & & (7.65) & (12.05) & & & (11.60) & $(13.43)$ & & & $(54.11)$ & (34.52) & & \\
\hline & Low & 6.10 & 10.49 & -2.57 & 0.0106 & 6.63 & 11.09 & -1.81 & 0.0448 & 8.19 & 24.44 & -1.82 & 0.0443 \\
\hline & & (3.06) & (3.87) & & & $(4.12)$ & $(5.75)$ & & & (3.54) & (24.92) & & \\
\hline & High & 13.10 & 15.81 & -0.58 & 0.2855 & 7.05 & 14.33 & -2.08 & 0.0277 & -3.43 & 4.84 & -1.82 & 0.0441 \\
\hline & & $(8.50)$ & (10.49) & & & (6.64) & (7.68) & & & (11.23) & (7.29) & & \\
\hline
\end{tabular}

\section{Preference for home bedding is lost}

We used a Y maze apparatus to characterize the effects of repetitive TBI on olfactory related behaviors (Online Resource 2 and Fig. 6a). Although both control $(\mathrm{N}=5)$ and repetitive TBI mice ( $\mathrm{N}=6)$ displayed a preference for home bedding (Fig. 6b), repetitive TBI mice exhibited longer duration in new bedding versus control mice. Unpaired t-test revealed that the amount of time or duration spent in the closed arm with home bedding differed slightly between groups but did not reach statistical significance (Fig. 6c).

\section{Discussion}

Olfactory dysfunction is an indicator of dementia risk, however whether it is present in individuals with a history of repetitive TBI has yet to be fully elucidated. As conventional neuroimaging fails to reveal underlying symptoms and abnormalities [25], we have demonstrated the structural and functional changes in the olfactory system using a repetitive TBI mouse model. Particularly, acute evaluation (1 week) after injury in repetitive TBI animals showed evident pathology in olfactory bulb associated areas. In almost all regions, not only did repetitive TBI mice show greater tauopathy compared to sham injury mice; areas displaying the greatest increase in tauopathy (AOL, GrO, and DTT) were also associated with cell size shrinkage or neuronal atrophy. Interestingly, the olfactory bulb, a region anatomically larger and possibly more vulnerable to injury in mice, was not prone to pathology. Similar pathological 
sequelae have been reported in other repetitive TBI models showing damage in rostral areas (optic tract, interior olfactory nuclei, lateral olfactory tract) after 3 repetitive TBI events [13]. Rather, it appears that regions directly caudal, adjoining the olfactory bulb, may be more vulnerable to cellular damage.

Studies have shown measured neuropathological consequences considerably after repetitive TBI events. For example, Mouzon et al. [15] were observed persistent white matter loss and Corpus Callosum thinning up till 12 months after repetitive TBI injury. Furthermore, the same study demonstrated that any history, even a single mTBI event, may be significantly injurious and could be correlated with cognitive decline or neurobehavioral differences [15]. In this regard, cell shrinkage, a characteristic of programmed cell death or apoptosis, may be an indicator of long-term cognitive impairments and reduced olfactory capacity. Whether the pathological sequelae in the olfactory system will persist and evolve post $\mathrm{mTBI}$ in the olfactory system remains to be clarified.

In this study, repetitive TBI produced subtle deficits in olfactory performance as measured in the y-maze task. The discrimination index showed that both $\mathrm{mTBI}$ and control mice displayed an overall stronger preference for home bedding than new bedding. Although TBI mice frequented their home bedding more than new bedding, they explored new bedding for longer than control mice. In addition, equivalent activity levels and velocity between control and mTBI mice showed that the loss of preference for home bedding in mTBI mice was not due to sensorimotor disturbances (Online Resource S1). This data suggests that the subtle loss of preference for home bedding in mTBI mice was due to a loss in olfactory discrimination.

The subtle differences in olfactory performance between mTBI and control mice is in alignment with the effects of repeated mTBI in humans. With a recent study that examined the olfactory functionality of former NFL players, former NFL players who experienced repetitive head impacts showed a lower performance in the Brief Smell Identification Test (B-SIT) compared to healthy controls. However, the majority of subjects ( 94\%) performed within healthy olfactory standards [11]. Although football players may experience, on average, more than 1000 subconcussive events per playing season, olfactory performance remains to be a subtle factor in describing brain injury. Similar results were obtained in analyses measuring visuospatial scanning speed, visuomotor processing speed, visuospatial construction, and verbal memory of NFL players compared to healthy controls [17]. Subtle olfactory differences may be difficult to detect with the current behavioral assessments used on human mTBI patients, and thus warrants further attention.

To overcome this weakness in behavioral assessments of olfactory functionality, here we used EEG recordings of repetitive TBI mice to detect olfactory dysfunction in repetitive TBI patients. Concerning the background resting-state EEG, our results showed that slow oscillation, especially the delta band oscillation, at the olfactory bulb was significantly decreased in the repetitive TBI mouse models. Also known as respiratory rhythms, olfactory slow oscillations are commonly observed in the mouse olfactory bulb, primary olfactory cortices, frontal cortex, and hippocampus and are synched to the respiratory cycle $[26,27]$. Even though there are not many previous studies concerning the olfactory delta oscillation, a recent study proposed that respiration-coupled slow oscillation activities play a major role in transferring information between olfactory-related brain regions to high-order brain regions [28], which suggest that weakened resting-state delta oscillation could reflect alterations in internal processing which thus causes impaired olfactory perception.

Furthermore, our data suggests a significant reduction in the resting state cross-frequency coupling (CFC) between the delta phase and beta/low gamma amplitude in repetitive TBI mouse models. Massive interconnected parallel processes in the brain require coordination of information between areas, and this often appears as CFCs $[29,30]$. Our finding is in line with previous studies that found a reduction of functional connectivity strength within the delta-beta and delta-gamma1 frequency pairs in the frontal brain region of mTBI human patients [31]. Recent studies have also been reporting the modulation of high frequency oscillations, such as local beta rhythm and gamma rhythms, by slow rhythms in the OB [27] and other higher order brain regions [32, 33], which suggests that the impaired resting state delta power could be the cause of the decreased CFC between delta and beta/low gamma band frequencies in repetitive TBI mouse models.

The present study also used the olfactory oddball paradigm to measure direct brain responses to odor stimuli. Oddball paradigms are frequently used to study sensory discrimination by comparing event-related potentials (ERP) or event-related spectral power (ERSP) from a standard stimulus with high probability to a deviant (oddball) stimulus with low probability. Typically, oddball stimuli elicit stronger responses in a correlational way of discriminability, thus, this paradigm has been widely used in conscious patients, infants, or animals that have limited expression in their language or motion. Here, using the olfactory odd-ball paradigm, we measured LFPs in the olfactory-bulb (OB), which serves as the direct receiver of olfactory sensory signals in the mouse brain, and other higher-order regions, such as the prefrontal cortex and hippocampus.

Regarding both the resting state LFP and ERSP during the olfactory oddball paradigm, our data showed significant neural impairments in the OB, whereas the prefrontal cortex and hippocampus appeared to be not affected by the injury. This is in line with past studies that show weakened brain activities in olfaction-related brain regions in PD patients that showed reduced olfactory functioning [34]. Especially, the power of beta oscillations was observed to be decreased in the $\mathrm{OB}$. The beta oscillation is known to be one of the main oscillatory rhythms linked to odor processing, commonly observed at the OB. The beta oscillation is reported to be strongly elicited by aversive odors with high volatility $[35,36]$. Also, past studies have shown enhanced beta power induced by repeated exposure to the same series of odorants [37], which suggests that the beta oscillation is closely linked to odor recognition and classification. Thus, decreased beta power in the OB during olfactory oddball tasks show impaired functionality in these specific areas of odor perception.

In conclusion, we show that after repetitive TBI events, olfactory related areas demonstrate abnormal electrophysiological changes, as well as increased tauopathy and decreased cell size. Tauphosphorylation causes cellular dysfunction resulting in decreased cell size associated with altered pathophysiology in olfactory related areas. These findings demonstrate that a history of repetitive TBI increases pathophysiology which may serve as a criterion of post-TBI neurodegenerative diseases such as chronic traumatic encephalography and dementia [38]. Our work further suggests that electrophysiological neural data can aid in revealing the olfactory impairment frequently observed in repetitive TBI, and the possible progression of brain damage in patients. Given the subtle behavioral changes observed during odor perception tests, patients with a history of repetitive TBI may be unaware of their condition. Without the use of verbal or behavioral examinations, early diagnosis of anosmia by its related EEG responses may be a more objective method in measuring brain damage.

Page 6/13 


\section{Abbreviations}

Traumatic brain injury (TBI); Anterior Olfactory Nucleus (AON); Pyriform Cortex (PC); Tenia Tecta (TT); Olfactory Tubercle (OT); Lateral Anterior Olfactory Nucleus (AOL); Granule Layer Olfactory Bulb (GrO); Dorsal Tenia Tecta (DTT); electroencephalography (EEG); chronic traumatic encephalopathy (CTE); Alzheimer's disease (AD); repetitive mild-TBI (mTBI); controlled cortical injury (CCI); blast-induced TBI (bTBI); fluid percussion injury (FPI); closed/head impact model of engineered rotational acceleration (CHIMERA); phosphate buffer saline (PBS); modulation index (MI); event-related spectral perturbation (ERSP); cross-frequency coupling (CFC); Olfactory Bulb (OB); event-related potentials (ERP).

\section{Declarations}

\section{Funding}

This study was supported by the Brain Research Program through Korean National Research Foundation (NRF-2016M3C7A1904233, NRF2018M3C7A1056894, NRF-2020M3E5D9079742, and 2017R1A2B3012659), and grants from Korea Institute of Science and Technology (2E30320, 2E30762).

\section{Competing Interest}

The authors declare that they have no competing interests in this section.

\section{Availability of data and materials}

The datasets generated and analyzed during the current study are available from the corresponding author on reasonable request.

\section{Author contributions}

YH.Y., Y.S., HJ.I., and H.-B.H were involved in experimentation, data curation and analysis; H.R. and J.H.C. were involved in the supervision of the study; YH.Y., Y.S., H.-B.H., J.H.C., and H.R. wrote the manuscript.

\section{Acknowledgements}

The authors thank Dr.Chaebin Kim for his assistance in preparing supplemental data video image and Jeungeun Kum for instructions on using the olfactometer used for the olfactory oddball paradigm.

\section{Ethics approval and consent to participate}

All procedures including surgery and experiment were followed by Korean Animal and Plant Quarantine Agency Publication No. 12512, partial amendment 2014, conforming to NIH guidelines (NIH Publication No. 86-23, revised 1985). All the procedures were approved by the Institutional Animal Care and Use Committee (IACUC) of Korea Institute of Science and Technology (KIST) (AP-2014L7002) according to international standards and guidelines.

\section{Consent to participate}

Not applicable

\section{Consent for publication}

Not applicable.

\section{References}

1. Faul M, Coronado V (2015) Epidemiology of traumatic brain injury. Handbook of clinical neurology 127: 3-13

2. Kaur P, Sharma S (2018) Recent Advances in Pathophysiology of Traumatic Brain Injury. Curr Neuropharmacol 16: 1224-1238. DOI $10.2174 / 1570159 \times 15666170613083606$

3. Najem D, Rennie K, Ribecco-Lutkiewicz M, Ly D, Haukenfrers J, Liu Q, Nzau M, Fraser DD, Bani-Yaghoub M (2018) Traumatic brain injury: classification, models, and markers. Biochem Cell Biol 96: 391-406. DOI 10.1139/bcb-2016-0160

4. Callahan CD, Hinkebein JH (2002) Assessment of anosmia after traumatic brain injury: performance characteristics of the University of Pennsylvania Smell Identification Test. The Journal of head trauma rehabilitation 17: 251-256

5. Pavlovic D, Pekic S, Stojanovic M, Popovic V (2019) Traumatic brain injury: neuropathological, neurocognitive and neurobehavioral sequelae. Pituitary 22: 270-282. DOI 10.1007/s11102-019-00957-9

6. Steuer E, Schaefer ML, Belluscio L (2014) Using the olfactory system as an in vivo model to study traumatic brain injury and repair. J Neurotrauma 31: 1277-1291. DOI 10.1089/neu.2013.3296 
7. Ng SY, Lee AYW (2019) Traumatic Brain Injuries: Pathophysiology and Potential Therapeutic Targets. Front Cell Neurosci 13: 528. DOI $10.3389 /$ fncel.2019.00528

8. Petraglia AL, Plog BA, Dayawansa S, Chen M, Dashnaw ML, Czerniecka K, Walker CT, Viterise T, Hyrien O, lliff JJ, et al. (2014) The spectrum of neurobehavioral sequelae after repetitive mild traumatic brain injury: a novel mouse model of chronic traumatic encephalopathy. J Neurotrauma 31: 12111224. DOI 10.1089/neu.2013.3255

9. Schneider T, Przewłocki R (2005) Behavioral alterations in rats prenatally exposed to valproic acid: animal model of autism. Neuropsychopharmacology 30: 80-89. DOI 10.1038/sj.npp.1300518

10. Fullard ME, Morley JF, Duda JE (2017) Olfactory dysfunction as an early biomarker in Parkinson's disease. Neuroscience bulletin 33: 515-525

11. Alosco ML, Jarnagin J, Tripodis Y, Platt M, Martin B, Chaisson CE, Baugh CM, Fritts NG, Cantu RC, Stern RA (2017) Olfactory function and associated clinical correlates in former national football league players. Journal of neurotrauma 34: 772-780

12. Broussard JI, Acion L, De Jesús-Cortés H, Yin T, Britt JK, Salas R, Costa-Mattioli M, Robertson C, Pieper AA, Arciniegas DB (2018) Repeated mild traumatic brain injury produces neuroinflammation, anxiety-like behaviour and impaired spatial memory in mice. Brain injury 32: 113-122

13. Creeley CE, Wozniak DF, Bayly PV, Olney JW, Lewis LM (2004) Multiple episodes of mild traumatic brain injury result in impaired cognitive performance in mice. Academic Emergency Medicine 11: 809-819

14. Hayes JP, Logue MW, Sadeh N, Spielberg JM, Verfaellie M, Hayes SM, Reagan A, Salat DH, Wolf EJ, McGlinchey RE, et al. (2017) Mild traumatic brain injury is associated with reduced cortical thickness in those at risk for Alzheimer's disease. Brain 140: 813-825. DOI 10.1093/brain/aww344

15. Mouzon BC, Bachmeier C, Ferro A, Ojo JO, Crynen G, Acker CM, Davies P, Mullan M, Stewart W, Crawford F (2014) Chronic neuropathological and neurobehavioral changes in a repetitive mild traumatic brain injury model. Ann Neurol 75: 241-254. DOI 10.1002/ana.24064

16. Xu L, Nguyen JV, Lehar M, Menon A, Rha E, Arena J, Ryu J, Marsh-Armstrong N, Marmarou CR, Koliatsos VE (2016) Repetitive mild traumatic brain injury with impact acceleration in the mouse: Multifocal axonopathy, neuroinflammation, and neurodegeneration in the visual system. Exp Neurol 275 Pt 3 : 436-449. DOI 10.1016/j.expneurol.2014.11.004

17. Raji CA, Tarzwell R, Pavel D, Schneider H, Uszler M, Thornton J, van Lierop M, Cohen P, Amen DG, Henderson T (2014) Clinical utility of SPECT neuroimaging in the diagnosis and treatment of traumatic brain injury: a systematic review. PLoS One 9: e91088. DOI 10.1371/journal.pone.0091088

18. Lehmkuhl AM, Dirr ER, Fleming SM (2014) Olfactory assays for mouse models of neurodegenerative disease. J Vis Exp: e51804. D0I 10.3791/51804

19. Yang M, Crawley JN (2009) Simple behavioral assessment of mouse olfaction. Curr Protoc Neurosci Chapter 8: Unit 8.24. DOI $10.1002 / 0471142301 . n s 0824 s 48$

20. Kum J, Kim JW, Braubach O, Ha JG, Cho HJ, Kim CH, Han HB, Choi JH, Yoon JH (2019) Neural Dynamics of Olfactory Perception: Low- and HighFrequency Modulations of Local Field Potential Spectra in Mice Revealed by an Oddball Stimulus. Front Neurosci 13: 478. DOI 10.3389/fnins.2019.00478

21. Mychasiuk R, Farran A, Angoa-Perez M, Briggs D, Kuhn D, Esser MJ (2014) A novel model of mild traumatic brain injury for juvenile rats. J Vis Exp. DOI $10.3791 / 51820$

22. Seo JS, Lee S, Shin JY, Hwang YJ, Cho H, Yoo SK, Kim Y, Lim S, Kim YK, Hwang EM, et al. (2017) Transcriptome analyses of chronic traumatic encephalopathy show alterations in protein phosphatase expression associated with tauopathy. Exp Mol Med 49: e333. DOI 10.1038/emm.2017.56

23. Paxinos G, and Charles Watson. (2006) The rat brain in stereotaxic coordinates: hard cover edition. Elsevier

24. Tort AB, Komorowski R, Eichenbaum H, Kopell N (2010) Measuring phase-amplitude coupling between neuronal oscillations of different frequencies. J Neurophysiol 104: 1195-1210. DOI 10.1152/jn.00106.2010

25. Johnson VE, Stewart W, Arena JD, Smith DH (2017) Traumatic brain injury as a trigger of neurodegeneration. Neurodegenerative diseases: $383-400$

26. Kocsis B, Pittman-Polletta BR, Roy A (2018) Respiration-coupled rhythms in prefrontal cortex: beyond if, to when, how, and why. Brain Struct Funct 223: 11-16. DOI 10.1007/s00429-017-1587-8

27. Lockmann ALV, Tort ABL (2018) Nasal respiration entrains delta-frequency oscillations in the prefrontal cortex and hippocampus of rodents. Brain Struct Funct 223: 1-3. DOI 10.1007/s00429-017-1573-1

28. Lockmann AL, Laplagne DA, Leão RN, Tort AB (2016) A Respiration-Coupled Rhythm in the Rat Hippocampus Independent of Theta and Slow Oscillations. J Neurosci 36: 5338-5352. DOI 10.1523/jneurosci.3452-15.2016

29. Fries P (2005) A mechanism for cognitive dynamics: neuronal communication through neuronal coherence. Trends in cognitive sciences 9: 474-480 
30. Lisman J, Buzsáki G (2008) A neural coding scheme formed by the combined function of gamma and theta oscillations. Schizophr Bull 34: $974-980$. DOI 10.1093/schbul/sbn060

31. Antonakakis M, Dimitriadis SI, Zervakis M, Micheloyannis S, Rezaie R, Babajani-Feremi A, Zouridakis G, Papanicolaou AC (2016) Altered crossfrequency coupling in resting-state MEG after mild traumatic brain injury. International Journal of Psychophysiology 102: 1-11

32. Biskamp J, Bartos M, Sauer J-F (2017) Organization of prefrontal network activity by respiration-related oscillations. Scientific reports 7: 1-11

33. Zhong W, Ciatipis M, Wolfenstetter T, Jessberger J, Müller C, Ponsel S, Yanovsky Y, Brankačk J, Tort ABL, Draguhn A (2017) Selective entrainment of gamma subbands by different slow network oscillations. Proc Natl Acad Sci U S A 114: 4519-4524. DOI 10.1073/pnas.1617249114

34. Baba T, Takeda A, Kikuchi A, Nishio Y, Hosokai Y, Hirayama K, Hasegawa T, Sugeno N, Suzuki K, Mori E (2011) Association of olfactory dysfunction and brain. Metabolism in Parkinson's disease. Movement Disorders 26: 621-628

35. Vanderwolf $\mathrm{CH}$, Zibrowski EM (2001) Pyriform cortex beta-waves: odor-specific sensitization following repeated olfactory stimulation. Brain Res 892: 301-308. DOI 10.1016/s0006-8993(00)03263-7

36. Zibrowski EM, Hoh TE, Vanderwolf CH (1998) Fast wave activity in the rat rhinencephalon: elicitation by the odors of phytochemicals, organic solvents, and a rodent predator. Brain Res 800: 207-215. DOI 10.1016/s0006-8993(98)00494-6

37. Vanderwolf CH, Zibrowski EM, Wakarchuk D (2002) The ability of various chemicals to elicit olfactory beta-waves in the pyriform cortex of meadow voles (Microtus pennsylvanicus) and laboratory rats (Rattus norvegicus). Brain Res 924: 151-158. DOI 10.1016/s0006-8993(01)03225-5

38. DeKosky ST, Asken BM (2017) Injury cascades in TBI-related neurodegeneration. Brain injury 31: 1177-1182

\section{Figures}

a

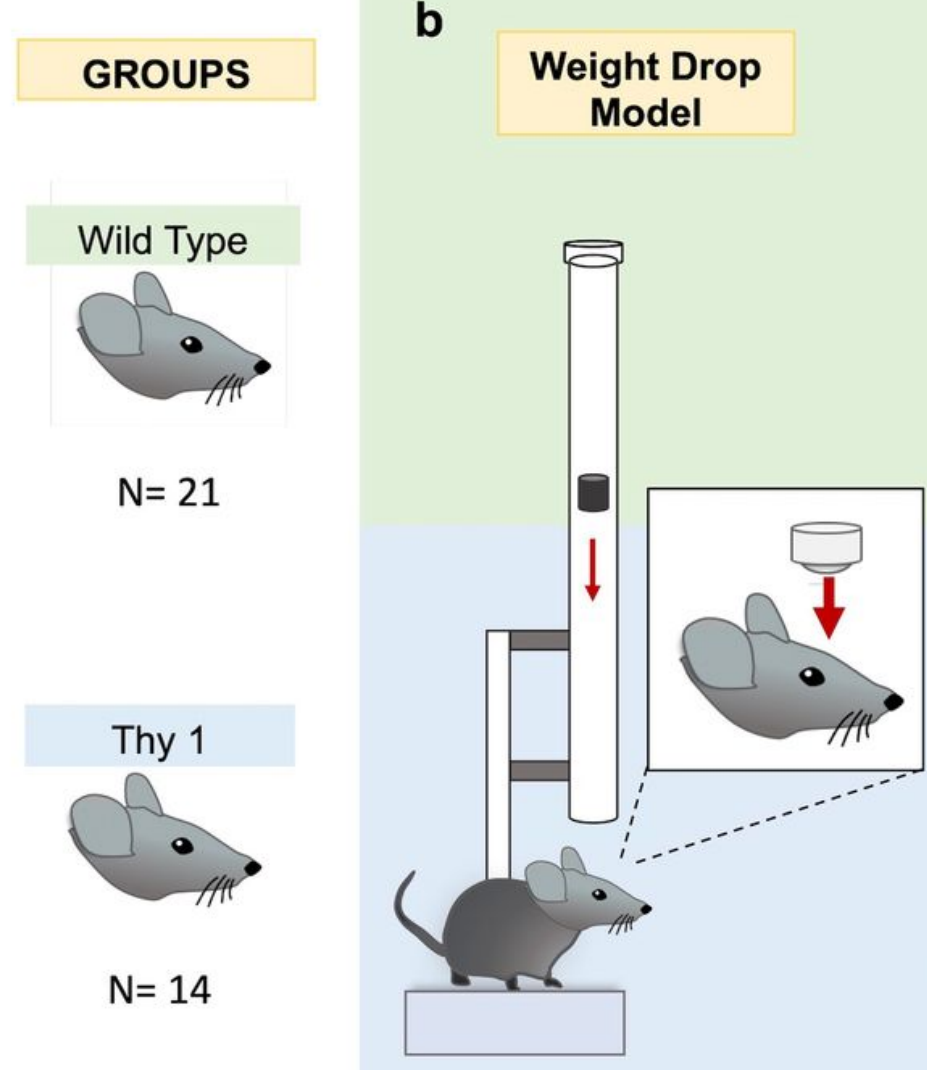

C

Pathology

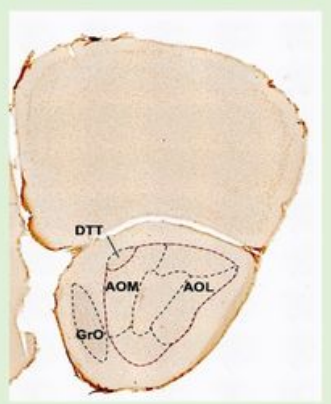

d

Behavior

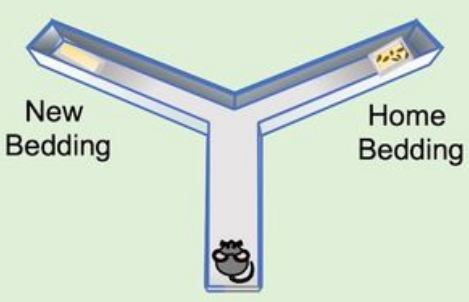

Start

e

Olfactory Oddball Paradigm

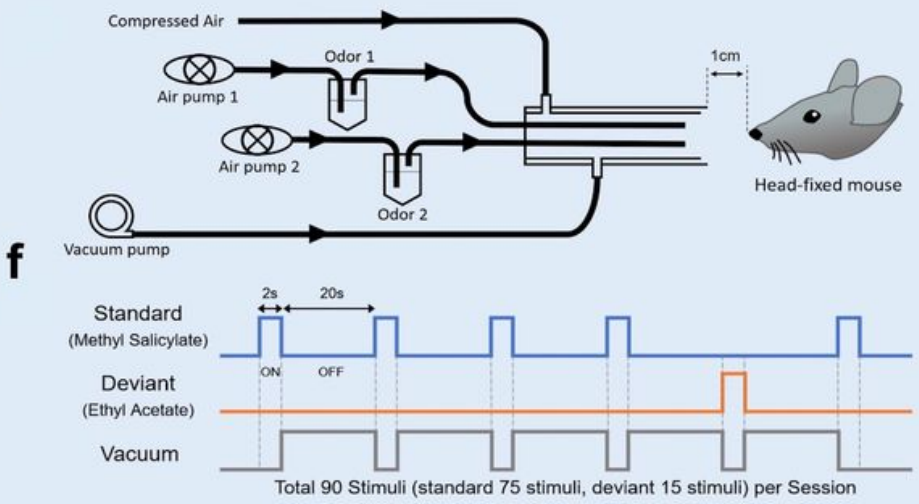

Figure 1

Weight-drop model of traumatic brain injury (TBI) and experiment workflows. a Wild type (WT) and Thy 1. b Mice experienced 5 weight drop induced TBI events. c Brain tissue was analyzed from postmortem WT mice ( $n=5$ control, $n=5$ TBI). d Living WT mice underwent $Y$ maze behavioral testing ( $n=5$ control, 
$\mathrm{n}=6 \mathrm{TBI}$ ). e A simplified diagram of the olfactometer used to deliver the two odor stimuli. Odor-evoked electrophysiology data of Thy 1 mice was collected using a two-odor olfactory oddball paradigm ( $n=8$ control, $n=9$ TBI). f Schematic overview of the two-odor olfactory oddball paradigm.

a

Central Olfactory System

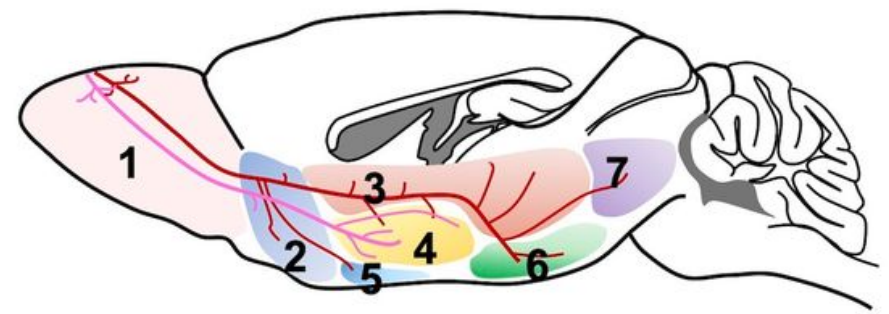

1. Olfactory Bulb

2. AON

3. $\mathrm{PC}$

4. OT b

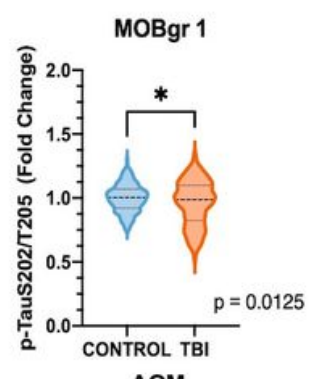

AOM

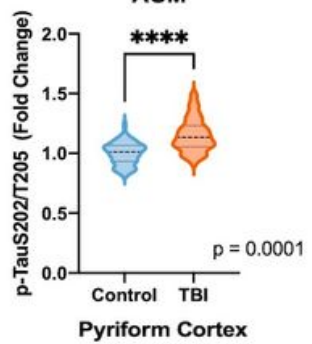

6. PLCo**

7. LEC
5. TT
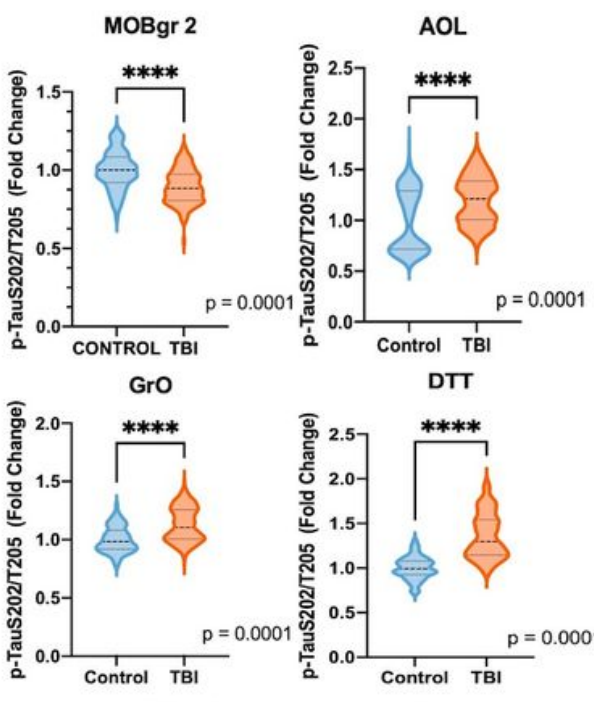

Olfactory Tubercle

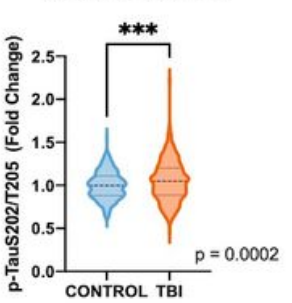

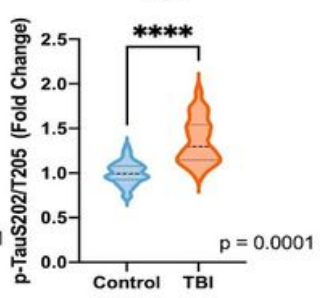

Entorhinal Cortex

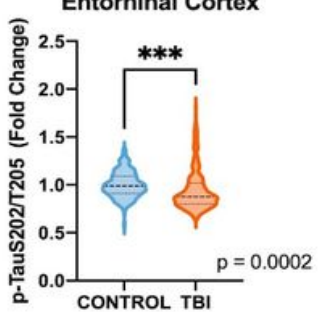

Figure 2

Olfactory bulb associated areas exhibit increased tauopathy in repetitive TBI. a A schematic diagram of the central olfactory system. Axonal projections from the olfactory bulb reach a number of areas including the Anterior Olfactory Nucleus (AON), Pyriform Cortex (PC), OIfactory Tubercle (OT), Tenia Tecta (TT), Lateral Entorhinal Cortex (LEC), and Cortical Amygdala (PLCo). **PLCo was unaccounted for analysis (Takeshi, 2014). b Densitometry analysis showed a significant increase in tauopathy in repetitive TBI mice [tissue, $\mathrm{N}=2-5$; cell counting, $\mathrm{n}=250$ (50 cells/tissue)] compared to control mice [tissue, $\mathrm{N}=3-5$; cell counting, $n=250$ (50 cells/tissue)]. Those regions included rostrally from the AON to the caudal regions of the TT $(p<0.05)$. Two regions of the Olfactory Bulb (MOBgr1 and MOBgr2) as well as the Entorhinal Cortex, did not reach statistical significance. Error bars indicate SEM, (controls, $\mathrm{N}=5$; repetitive TBI, $\mathrm{N}=5$ ). 
a

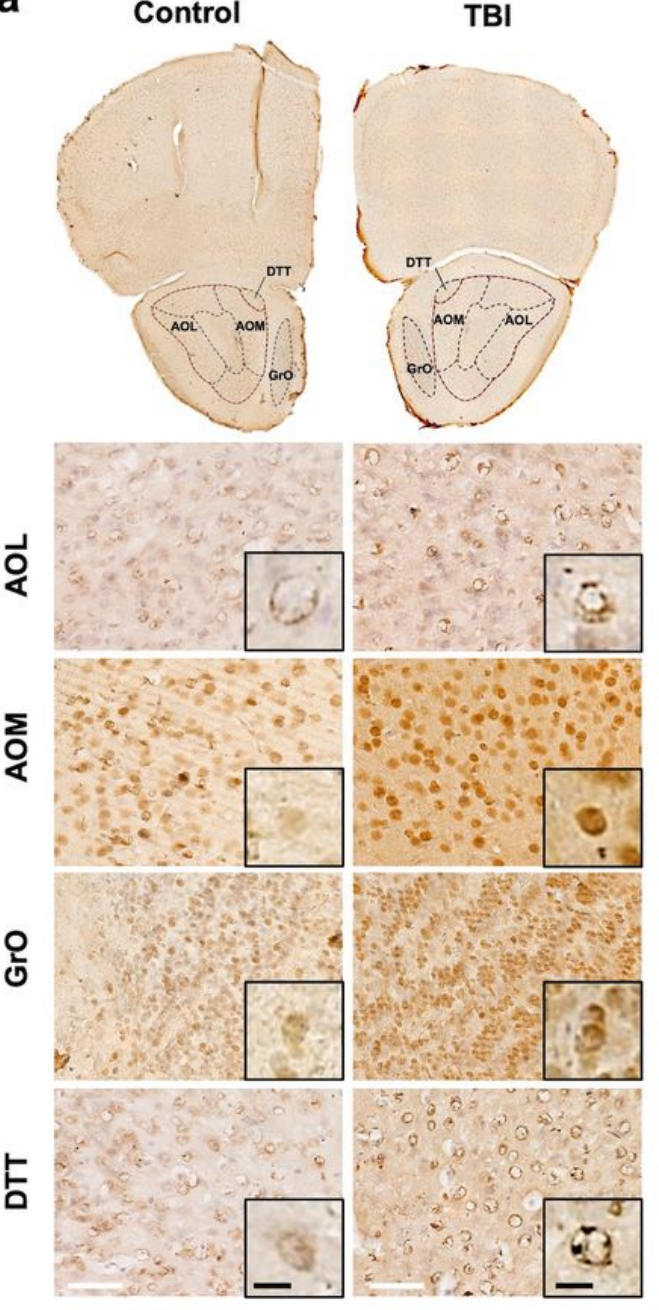

b
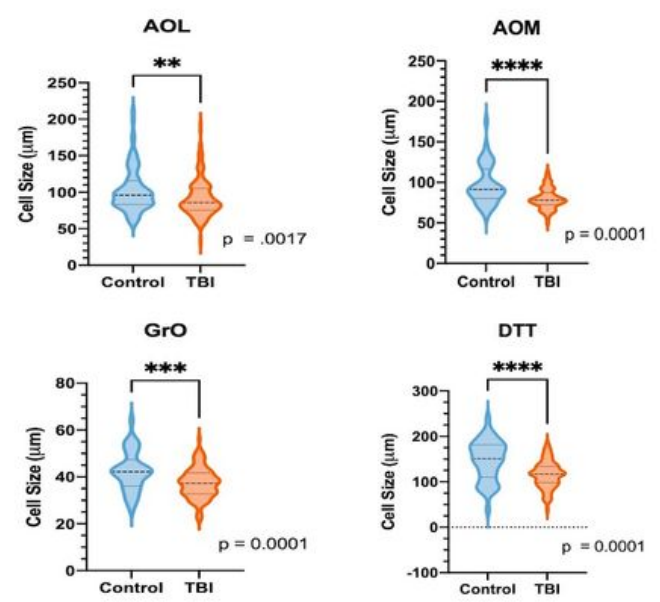

C
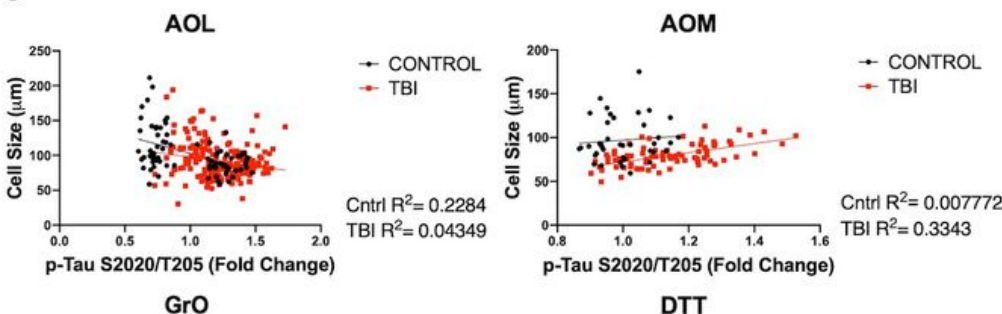

DTT
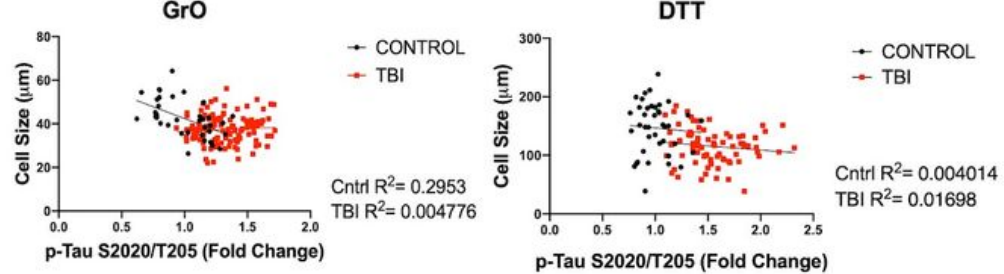

Figure 3

Tauopathy of olfactory bulb-associated areas is correlated with neuronal atrophy by repetitive TBI. a Tauopathy was increased and cell size was decreased

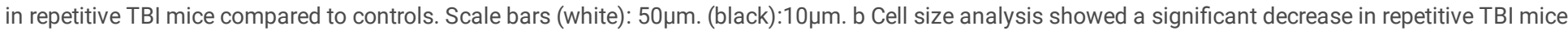
[tissue, $\mathrm{N}=2-5$; cell counting, $\mathrm{n}=100$ (20 cells/tissue)] compared to controls [tissue, $\mathrm{N}=3-5$; cell counting, $\mathrm{n}=100$ (20 cells/tissue)] in the AOL, AOM, GrO, and DTT. $p=.0017, p=0.0001$. $c$ Densitometry and Cell Size analysis exhibited a link between tauopathy and neuronal atrophy. AOL, GrO, and DTT regions exhibited a downward regression, (controls, $\mathrm{N}=5$; repetitive $\mathrm{TBI}, \mathrm{N}=5$ ). 

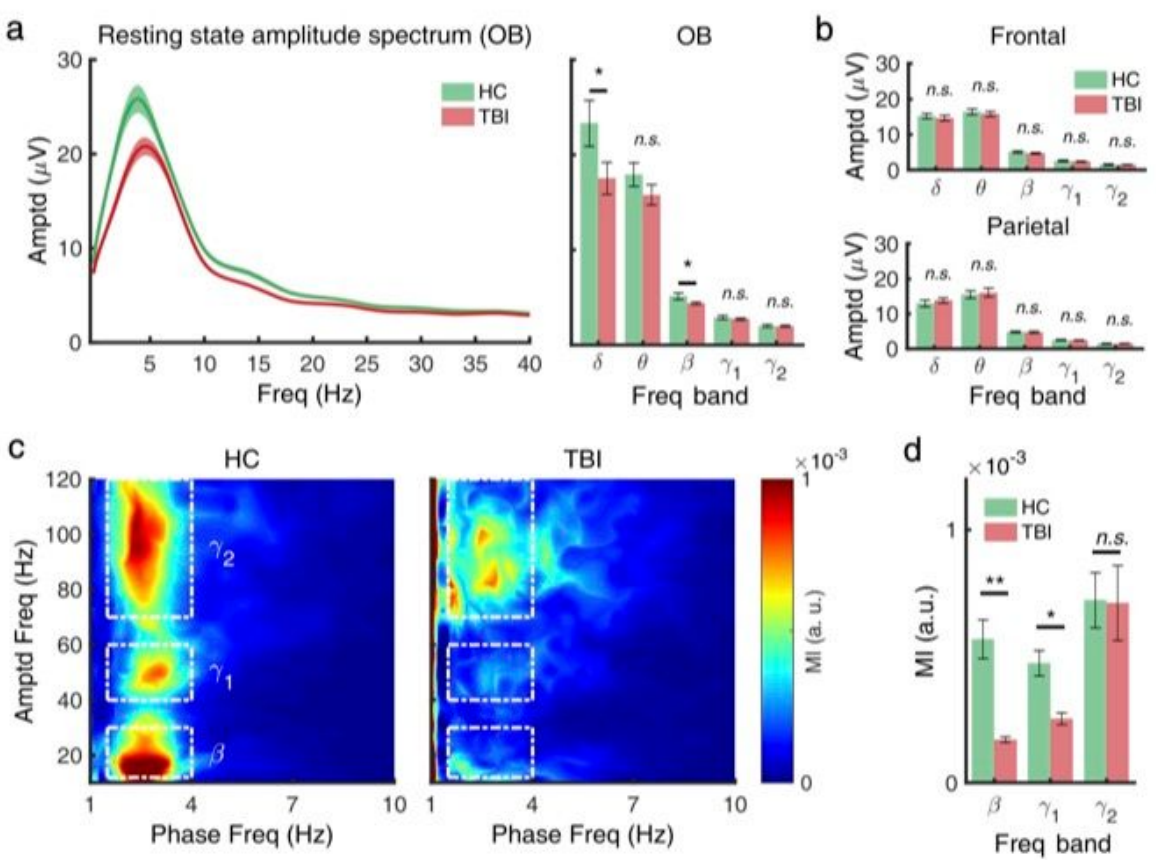

\section{Figure 4}

Olfactory bulb exhibits reduced delta oscillations and modulation index by repetitive TBI. a Resting state phase amplitude analysis in the olfactory bulb. There was a decrease $(p=0.0309)$ in resting state delta-band amplitude in TBI mouse models. $b$ There was no significant amplitude change observed at the Frontal and Parietal regions of the mouse brain. c Comodulogram comparing healthy and TBI mouse subjects' cross frequency coupling with delta bands. TBI mouse models showed reduced cross frequency coupling in delta-beta and delta-theta1 phase pairs. d Statistical analysis illustrating lowered MI of delta-beta $(p=0.0077)$, delta-theta $(p=0.0289)$ oscillation pairs of TBI mouse models.
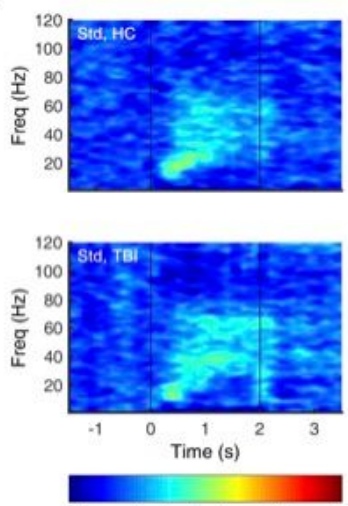

Amplitude change (\%)
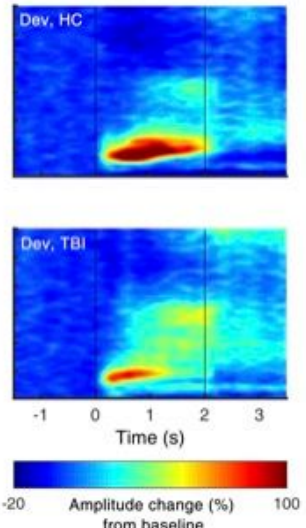
trom baseline b
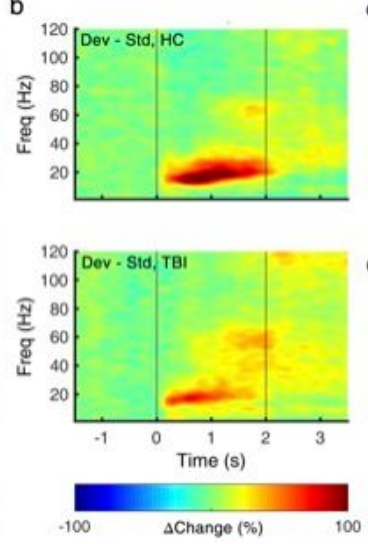

C

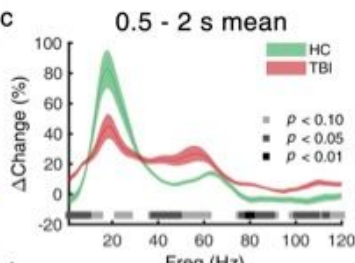

d

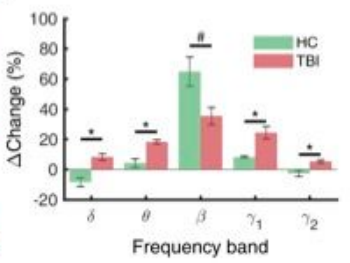


Response to deviant odors is attenuated by repetitive TBI mice. a Power spectrogram illustrating averaged ERSP for standard and deviant odors. Deviant odors elicit a stronger ERSP response, especially in beta band range. b Differential spectrogram of ERSP response. c Mean of amplitude difference during $0.5-2$ s time range. $d$ Statistical analysis of amplitude difference of each band range. Relative power of beta oscillation decreased $(p=0.0985)$, while other frequency ranges showed increased relative power $(p<0.05)$.

a

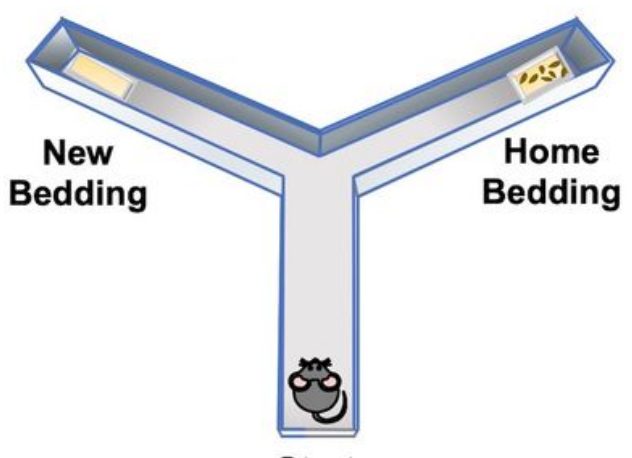

b

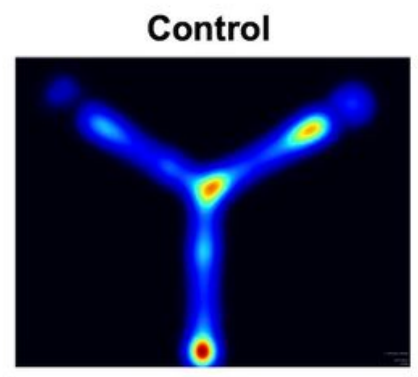

C

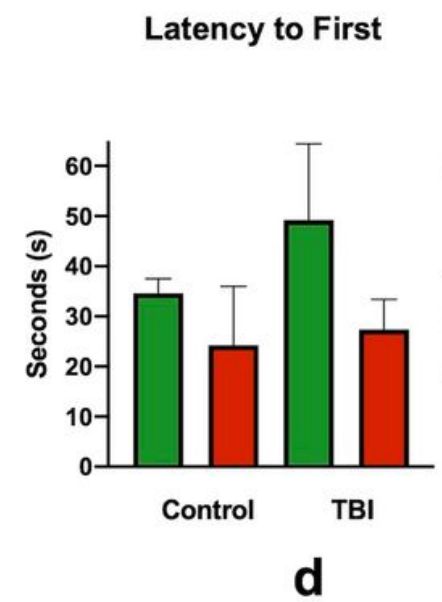

\section{New Bedding $\square$ Home Bedding}
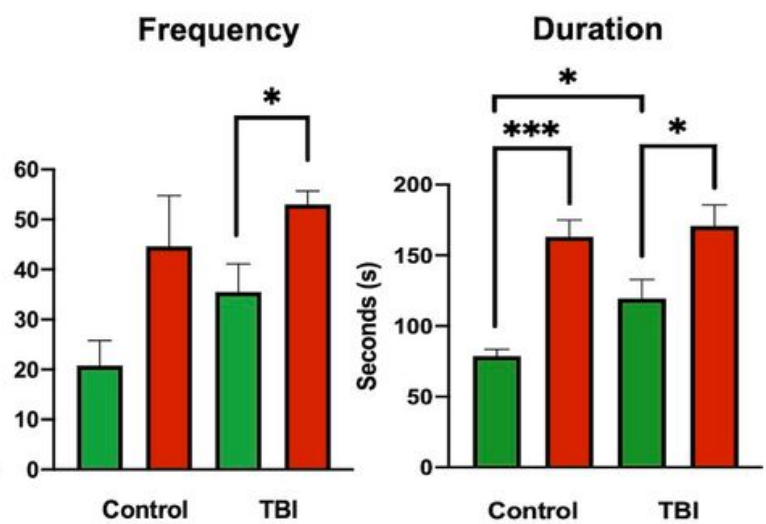

Discrimination Index

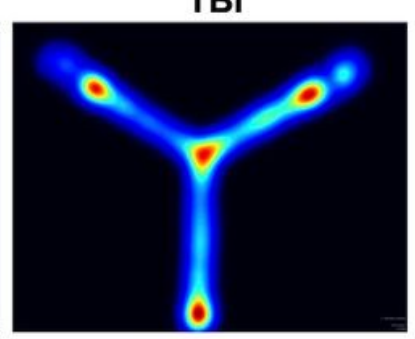

Duration (s)

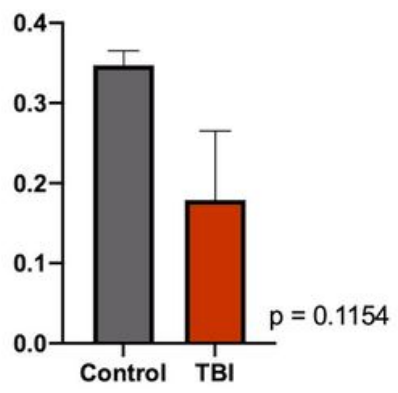

Frequency

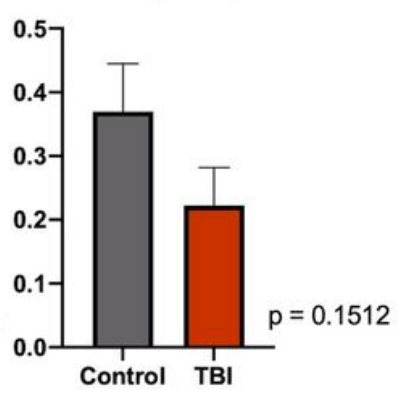

Figure 6

Repetitive TBI shows a reduced preference for home bedding. a Y maze apparatus and placement of new bedding and home bedding. $\mathrm{b}$ A representative heat map of $Y$ maze trials show an increased frequency in TBI mice in the arm with new bedding. c Control and TBI mice display alterations of latency to first, frequency, and duration. TBI mice show greater frequency for home bedding $(p=0.0189)$ as well as a greater duration for new bedding ( $p=0.0276)$ compared to controls. $d$ Discrimination Index for duration and frequency shows that control mice have a greater preference for home bedding $(p=0.1154, p$ $=0.1512$ ) [control, $\mathrm{N}=5 ; \mathrm{TBI}, \mathrm{N}=6]$.

\section{Supplementary Files}

This is a list of supplementary files associated with this preprint. Click to download.

- SupplementalFigureS1.jpg

- SupplementaryInformationJMolecularMedicine.docx 